\title{
Unusual Case of Subcutaneous Angiosarcoma Metastatic to the Ovary
}

\author{
David CANTÚ DE LEÓN, ${ }^{1}$ Delia PÉREZ MONTIEL, ${ }^{2}$ José CHANONA VILCHIS ${ }^{2}$
}

Departments of ${ }^{1}$ Surgical Oncology and ${ }^{2}$ Pathology, Instituto Nacional de Cancerologia, Mexico City, Mexico

The ovaries are common sites for metastatic disease, however, the most frequent ones are carcinomas. Metastatic sarcomas are very rare in ovary and most of them arise from genital tract. We present the case of a 33-year-old woman with subcutaneous

Key words: ovary, metastatic sarcoma, angiosarcoma angiosarcoma who had metastatic disease to the ovary resulting in acute abdominal pain. Discussion of the case and a review of the literature are presented. (Pathology Oncology Research Vol 13, No $4,379-381)$

\section{Introduction}

Ovarian masses are common findings in general gynecological practice; approximately $5-10 \%$ of the ovarian malignances are diagnosed as metastatic tumors. ${ }^{14}$ It is known that colon cancer is the most frequent primary malignancy of non-genital origin that metastasizes to the ovary. ${ }^{6}$ Nevertheless, sarcomas can rarely metastasize to this organ. Genital sarcomas are the most common metastatic sarcomas and, infrequently, cases of gastrointestinal or urological sarcomas have been reported as metastatic tumor in the ovary. ${ }^{15}$ Cases originating from the soft tissue are extremely unusual.

\section{Case Report}

\section{Clinical history}

The patient is a 33-year-old Mexican woman; her past medical, surgical, gynecologic and family histories were all unremarkable. Her chief complaint was a slow growing tumor in the right buttock, which became abscessed three and a half years after appearance. The patient was submitted to surgical drainage of the abscess and an incisional biopsy was taken.

The diagnosis of subcutaneous angiosarcoma was made and the patient was sent to our institution for further evaluation and treatment; her admission was on January, 2004.

Received: Nov 3, 2005; accepted: Nov 20, 2007

Correspondence: David CANTÚ DE LEÓN, Instituto Nacional Cancerologia, Av San Fernando \#22 Col Seccion XVI. ZP 14080 Mexico City, México. Tel: 52-5525397170, fax: 525556280422. E-mail: dcantu@itesm.mx
Laboratory examinations, chest X-ray and an abdominopelvic CT scan were performed and revealed only a lesion on the buttock with normal abdominal and pelvic organs. On the physical examination the only remarkable finding was the scar of the previous surgery with evidence of local inflammatory response. Surgical excision of the lesion was performed on February 26, 2004 with clear surgical margins, and adjuvant radiotherapy of 64 Gy was given from May 3 to July 7, 2004.

The patient was followed each month and remained asymptomatic until October 29, 2004 when she presented to the emergency room for evaluation of abdominal pain lasting 2 days. Laboratory studies revealed hemoglobin 13.4 $\mathrm{g} / \mathrm{dl}$, white blood count $12000 / \mathrm{mm}^{3}$, hematocrit $39.4 \%$, 268,000 platelets. Liver function tests, glucose and tumor markers were within normal limits. Chest x-ray was normal. A pelvic CT scan revealed a solid and cystic left ovarian tumor measuring $7 \times 4 \mathrm{~cm}$ (Fig. 1). Abdominal physical

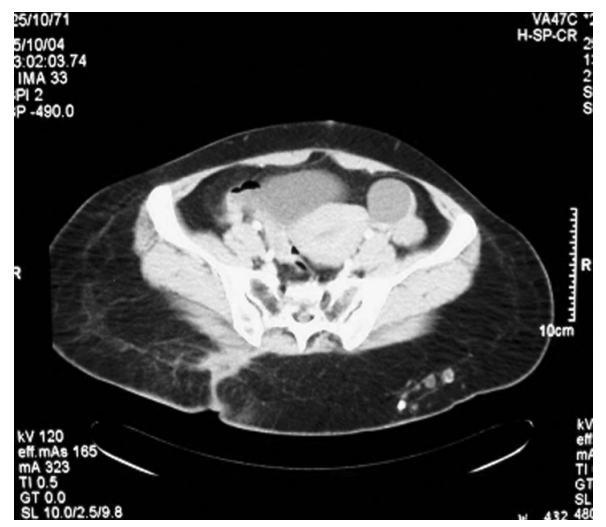

Figure 1. CT scan showing ovarian cyst mass without solid areas. 


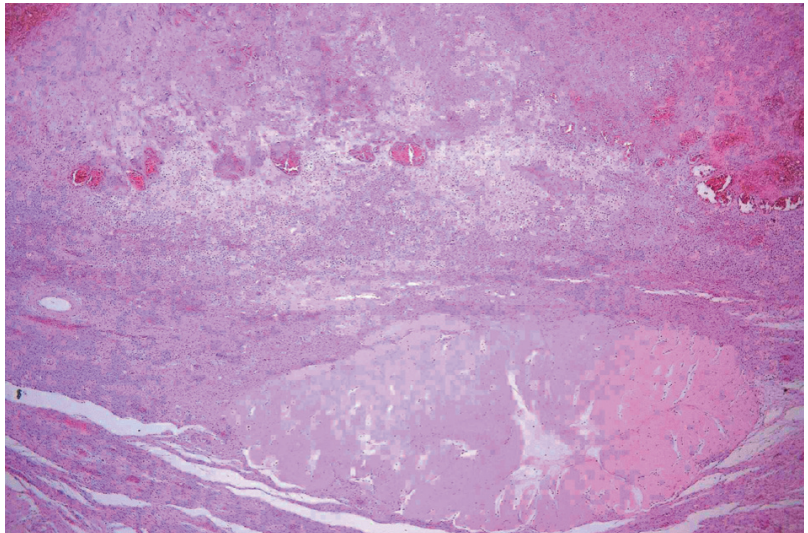

Figure 2. Metastatic ovarian angiosarcoma. The image shows residual ovary with hemorrhagic mass adjacent. (HE; x32)

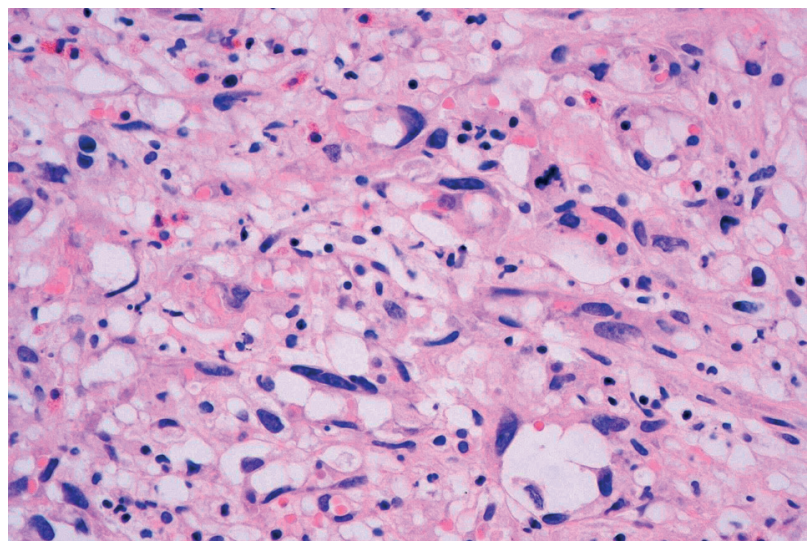

Figure 3. Hemorrhagic ovarian mass showing pleomorphic cells with intracytoplasmic lumens $(H E ; x 400)$. These cells are CD31-positive (insert: immunoperoxidase, $x 400$ )

examination revealed pain with positive rebound in the left iliac fossa, with normal peristalsis. On gynecologic examination a tender mass was palpated on the left iliac fossa, pain on cul-de-sac exacerbated with movement of the cervix.

An exploratory laparotomy was performed on the day of admission, with resection of the left adnexa and omentum that was adhered to the area. No ascites or other remarkable alterations were found. Final pathologic report was metastatic angiosarcoma within a simple ovarian cyst. Local radiotherapy and chemotherapy were proposed as adjuvant treatment but the patient refused, and she was placed under observation. She is alive but with local recurrence in the buttock and lung metastasis six months later.

\section{Pathologic findings}

Histopathologic study of the primary lesion in the buttock revealed a subcutaneous tumor exhibiting proliferation of atypical capillaries and anastomosing vascular channels. These structures were delineated by atypical endothelial cells. Other parts of the tumor showed solid areas populated by polygonal pleomorphic cells featuring intracytoplasmic lumens. The final diagnosis was subcutaneous high-grade angiosarcoma.

The ovarian lesion was an ill-defined hemorrhagic mass measuring $7 \times 5 \times 5 \mathrm{~cm}$. The cut surface showed residual ovary. Microscopically a hemorrhagic lesion with vascular channels delineated by atypical endothelial cells was observed (Fig. 2, and 3).

Immunohistochemical studies were performed on samples of the primary and metastatic tumors. The neoplastic cells in both neoplasias were positive for CD34, CD31 and vimentin, and negative for cytokeratin AE1/AE3, EMA, actin, desmin and S-100 protein (Fig. 4).

The buttock and ovarian masses were similar from the histopathologic and immunohistochemical points of view.

\section{Discussion}

Ovaries are common sites for metastatic disease. Extensive information about spread of carcinomas is available in the international literature. ${ }^{1,4-7}$ However, there is relatively little information concerning the spread and frequency of metastatic sarcomas to this organ. Thus, there may be difficulties in the diagnosis and treatment options for these patients.

Very few series of sarcomas metastatic to the ovary had been published, one of the most important being the review by Young and Scully ${ }^{3}$ in 1990, in which they describe 21 cases of metastatic sarcomas to the ovaries. It is noteworthy that the most common primary sites are genital organs, such as uterine corpus and cervix (11 of 21 cases), ${ }^{3}$ and the remainder comprises other organs such as the small intestine, stomach and bladder. In the mentioned series only one case was a large hemangiosarcoma probably originating from the heart, which was asymptomatic despite its large size, different from our

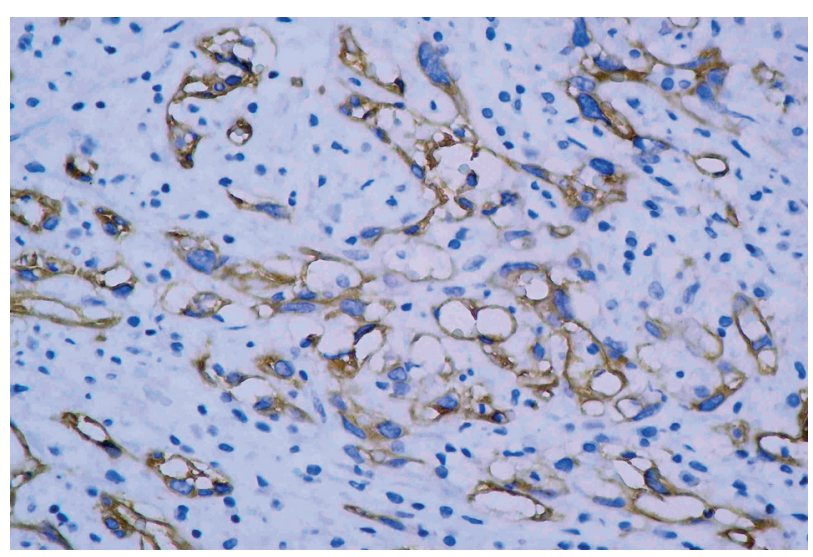

Figure 4. There cells are CD31-positive $(+400)$ 
case where the patient who complained of abdominal pain. Other primary sites included bone and anterior abdominal wall.

Survival of these patients is poor, ranging from $10-12 \%$ at 5 -years. ${ }^{10,16}$ This is basically because the tumor is inoperable at the moment of diagnosis as documented by Petru in his series of 82 cases. ${ }^{10}$ However, it is important to note that this series include only metastatic carcinomas.

Treatment must be focused on removing as much tumor as possible, ${ }^{11}$ recalling the metastatic nature of the disease and the poor prognosis involved.

In conclusion, past oncologic history of any patient with abdominal pain or abdominal mass is the most important clue for the diagnosis of a metastatic sarcoma to the ovary, even though the event is relatively uncommon. Establishing the diagnosis is easy, but diagnostic difficulties can be present occasionally due to the specific pathologic or clinical features.

\section{References}

1. Moore RG, Chung M, Granai CO, et al: Incidence of metastasis to the ovaries from nongenital tract primary tumors. Gynecol Oncol 93:87-89, 2004.

2. Ulbright TM, Roth LM, Stehman FB: Secondary ovarian neoplasia. A clinicopathologic study of 35 cases. Cancer 53:11641174, 1984.

3. Young RH, Scully RE: Sarcomas metastatic to the ovary: A report of 21 cases. Int J Gynecol Pathol 9:931-952, 1990.
4. Mazur MT, Hsueh S, Gersell DJ: Metastases to the female genital tract. Analysis of 325 cases. Cancer 53:1978-1984, 1984.

5. Fujiwara K, Ohishi Y, Koike H, et al: Clinical implications of metastases to the ovary. Gynecol Oncol 59:124-128, 1995.

6. Blamey S, McDermott F, Pihl E, et al: Ovarian involvement in adenocarcinoma of the colon and rectum. Surg Gynecol Obstet 153:42-44, 1981.

7. Demopoulos RI, Tuger L, Dubin N: Secondary ovarian carcinoma: a clinical and pathological evaluation. Int J Gynecol Pathol 6:166-175, 1987.

8. Pillay K, Essa AS, Chetty R: Borderline serous cystadenocarcinoma with coexistent angiosarcoma: an unusual form of ovarian carcinoma. Int J Surg Pathol 9:317-321, 2001.

9. Platt JS, Rogers SJ, Flynn CA, et al. Primary angiosarcoma of the ovary: A case report and review of the literature. Gynecol Oncol 73: 443-446, 1999.

10. Scully R. Young RH, Clement PB: Tumors of the ovary, maldeveloped gonads, fallopian tube and broad ligament. Armed Forces Institute of Pathology, 3rd series, 1998.

11. Petru E, Pickel H, Heydarfadai $M$, et al: Nongenital cancers metastatic to the ovary. Gynecol Oncol 44:83-86, 1992.

12. Yada-Hashimoto $N$, Yamamoto T, Kamiura $S$, et al: Metastatic ovarian tumors: A review of 64 cases. Gynecol Oncol 89:314317,2003

13. Shiromizu K, Kawana T, Sugase $M$, et al: Experience with the treatment of metastatic ovarian carcinoma. Arch Gynecol Obstet 243:111-114, 1988

14. Soloway I, Latour J, Young M: Krukenberg tumors of the ovary. Obstet Gynecol 8:636-638, 1956.

15. Eltabbakh $G H$, Belison JL, Biscotti CU: Osteosarcoma metastatic to the ovary: A case report and review of the literature. Int J Gynecol Pathol 16:76-78, 1997.

16. Webb M, Decker D, Mussey E: Cancer metastatic to the ovary. Obstet Gynecol 45:391-396, 1975. 